\title{
Diastereoselective Multicomponent Synthesis of Dihydropyridones with an Isocyanide Functionality
}

\author{
Monica Paravidino, ${ }^{\ddagger}$ Robin S. Bon,,${ }^{\ddagger}$ Rachel Scheffelaar, ${ }^{\ddagger}$ Danielle J. Vugts, ${ }^{\ddagger}$ Anass Znabet,,${ }^{\ddagger}$ Rob F. \\ Schmitz, ${ }^{\ddagger}$ Frans J. J. de Kanter, ${ }^{\ddagger}$ Martin Lutz, ${ }^{\S}$ Anthony L. Spek, ${ }^{\S}$ Marinus B. Groen ${ }^{\ddagger}$ and Romano V. A. \\ $\mathrm{Orru}^{\ddagger *}$ \\ Vrije Universiteit Amsterdam, Department of Chemistry, De Boelelaan 1083, 1081 HV Amsterdam, The Netherlands \\ and Bijvoet Center for Biomolecular Research, Crystal and Structural Chemistry Utrecht University, Padualaan 8, \\ 3584 CH Utrecht, The Netherlands \\ RECEIVED DATE (automatically inserted by publisher); E-mail: orru@few.vu.nl
}

\section{Supporting information}

\begin{abstract}
General Information: All reactions were carried out under an inert atmosphere of dry argon. Standard syringe techniques were applied for transfer of air sensitive reagents and dry solvents. Melting points were measured using a Stuart Scientific SMP3 melting point apparatus and are uncorrected. Infrared (IR) spectra were obtained from $\mathrm{CHCl}_{3}$ films on $\mathrm{NaCl}$ tablets (unless noted otherwise), using a Matteson Instuments 6030 Galaxy Series FT-IR spectrophotometer and wavelengths $(v)$ are reported in $\mathrm{cm}^{-1} .{ }^{1} \mathrm{H}$ and ${ }^{13} \mathrm{C}$ nuclear magnetic resonance (NMR) spectra were recorded on a Bruker Avance $400(400.13 \mathrm{MHz}$ and 100.61 MHz respectively), a Bruker Avance 250 (250.13 MHz and 62.90 MHz respectively) or a Bruker Avance 200 (200.13 MHz and $50.32 \mathrm{MHz}$ respectively) with chemical shifts $(\delta)$ reported in ppm downfield from tetramethylsilane. Peak assignment was also done with the aid of gs-COSY, gs-HMQC and gs-HMBC measurements. Assignment of relative stereochemistry was achieved using gs-NOESY measurements. MS and HRMS spectra data were recorded on a Finnigan Mat 900 spectrometer. Chromatographic purification refers to flash chromatography using the indicated solvent (mixture) and Baker 7024-02 silica gel $\left(40 \mu, 60 \AA\right.$ ). Thin Layer Chromatography was performed using silica plates from Merck (Kieselgel $60 \mathrm{~F}_{254}$ on aluminium with fluorescence indicator. Compounds on TLC were visualised by UV-detection. THF was dried and distilled from sodium benzophenone ketyl prior to use. Benzonitrile was dried with $\mathrm{MgSO}_{4}$ and then distilled from $\mathrm{P}_{2} \mathrm{O}_{5}$ under reduced pressure. Other commercially available reagents were used as purchased.
\end{abstract}

General Procedure I for the synthesis of dihydropyridones: All reactions were carried out at a concentration of $0.2 \mathrm{M}$ of diethyl methylphosphonate $1,0.24$ $\mathrm{M}$ of $n$-BuLi, $0.22 \mathrm{M}$ of nitrile, $0.22 \mathrm{M}$ of aldehyde and $0.22 \mathrm{M}$ of isocyanoacetate in dry THF. Always 1.0 mmol of the limiting reaction component (diethyl methylphosphonate 1) was used. 1.2 equiv. of $n$ - $\mathrm{BuLi}$ (1.6 M solution in hexane) were added at $-78{ }^{\circ} \mathrm{C}$ to a stirred solution of phosphonate in THF. After stirring at $-78{ }^{\circ} \mathrm{C}$ for $1.5 \mathrm{~h}$ the nitrile (1.1 equiv.) was added and the mixture was then stirred at $-78{ }^{\circ} \mathrm{C}$ for $45 \mathrm{~min}$., at $-40{ }^{\circ} \mathrm{C}$ for $1 \mathrm{~h}$ and at $-5{ }^{\circ} \mathrm{C}$ for 30 min. The aldehyde (1.1 equiv.) was added and, after stirring at $-5{ }^{\circ} \mathrm{C}$ for $30 \mathrm{~min}$., the mixture was allowed to warm to rt and stirred for $1.5 \mathrm{~h}$. Finally the isocyanoacetate (1.1 equiv.) was added and the mixture was stirred overnight. The reaction mixture was concentrated in vacuo and the crude product purified by column chromatography.

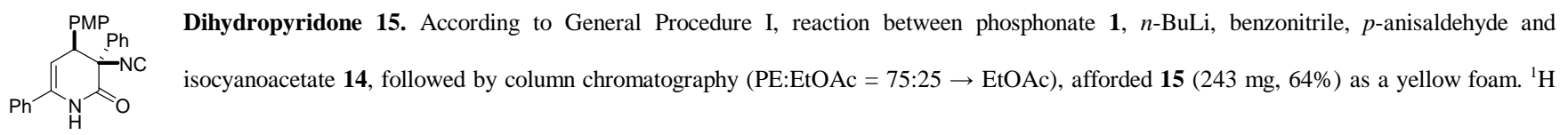

\footnotetext{
$\ddagger$ Vrije Universiteit Amsterdam

${ }^{\S}$ Utrecht university
} 
NMR (400 MHz, $\left.\mathrm{CDCl}_{3}\right): \delta(\mathrm{ppm}) 7.53(\mathrm{~s}, 1 \mathrm{H}), 7.47-7.34(\mathrm{~m}, 10 \mathrm{H}), 7.04(\mathrm{~d}, J=8.7 \mathrm{~Hz}, 2 \mathrm{H}), 6.77(\mathrm{~d}, J=8.7 \mathrm{~Hz}, 2 \mathrm{H}), 5.66(\mathrm{dd}, J=4.5,1.7 \mathrm{~Hz}, 1 \mathrm{H}), 4.25(\mathrm{~d}$, $J=4.4 \mathrm{~Hz}, 1 \mathrm{H}), 3.75(\mathrm{~s}, 3 \mathrm{H}) ;{ }^{13} \mathrm{C}$ NMR (50 MHz, $\left.\mathrm{CDCl}_{3}\right): \delta(\mathrm{ppm}) 165.8(\mathrm{C}), 162.8(\mathrm{C}), 160.0(\mathrm{C}), 137.3(\mathrm{C}), 135.8(\mathrm{C}), 133.9(\mathrm{C}), 130.9(2 \times \mathrm{CH}), 130.0$ $(\mathrm{CH}), 129.5(2 \times \mathrm{CH}), 129.4(\mathrm{CH}), 129.0(2 \times \mathrm{CH}), 127.8(\mathrm{C}), 126.8(2 \times \mathrm{CH}), 125.6(2 \times \mathrm{CH}), 114.3(2 \times \mathrm{CH}), 105.9(\mathrm{CH}), 71.1(\mathrm{C}), 55.7(\mathrm{CH}), 51.1(\mathrm{CH}) ; \mathrm{IR}$ (KBr): 2127 (m), 1708 (s), 1528 (s), 1472 (m), 1264 (m); HRMS (EI, $70 \mathrm{eV}$ ): calculated for $\mathrm{C}_{25} \mathrm{H}_{20} \mathrm{~N}_{2} \mathrm{O}_{2}\left(\mathrm{M}^{+}\right)$380.1525, found 380.1516; MS (EI, $\left.70 \mathrm{eV}\right)$ : m/z $(\%)=380(61)\left[\mathrm{M}^{+}\right], 236(100), 115(8), 104(10)$.

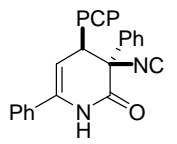

Dihydropyridone 18. According to General Procedure I, reaction between phosphonate 1, $n$-BuLi, benzonitrile, $p$-chlorobenzaldehyde and isocyanoacetate 14, followed by column chromatography ( $c$-hexane:EtOAc $=9: 1 \rightarrow 7: 3)$, afforded $18(250 \mathrm{mg}, 72 \%)$ as a yellow foam. ${ }^{1} \mathrm{H}$ NMR (250 MHz, $\left.\mathrm{CDCl}_{3}\right): \delta(\mathrm{ppm}) 7.61(\mathrm{~s}, 1 \mathrm{H}), 7.49-7.39(\mathrm{~m}, 10 \mathrm{H}), 7.23(\mathrm{~d}, J=8.5 \mathrm{~Hz}, 2 \mathrm{H}), 7.05(\mathrm{~d}, J=8.5 \mathrm{~Hz}, 2 \mathrm{H}), 5.64(\mathrm{dd}, J=4.1$, $1.3 \mathrm{~Hz}, 1 \mathrm{H}), 4.29(\mathrm{~d}, J=4.2 \mathrm{~Hz}, 1 \mathrm{H}) ;{ }^{13} \mathrm{C}$ NMR $\left(63 \mathrm{MHz}, \mathrm{CDCl}_{3}\right): \delta(\mathrm{ppm}) 165.0(\mathrm{C}), 162.8(\mathrm{C}), 137.5(\mathrm{C}), 134.8(\mathrm{C}), 134.3(\mathrm{C}), 134.1(\mathrm{C}), 133.3(\mathrm{C}), 130.6$ $(2 \times \mathrm{CH}), 129.7(\mathrm{CH}), 129.10(2 \times \mathrm{CH}), 129.07(\mathrm{CH}), 128.59(2 \times \mathrm{CH}), 128.57(2 \times \mathrm{CH}), 126.3(2 \times \mathrm{CH}), 125.2(2 \times \mathrm{CH}), 104.6(\mathrm{CH}), 70.3(\mathrm{C}), 50.9(\mathrm{CH}) ; \mathrm{IR}$ (neat): 2127 (w), 1699 (s), 1490 (m), 1092 (m), 1014 (m); HRMS (EI, $70 \mathrm{eV)}$ ) calculated for $\mathrm{C}_{24} \mathrm{H}_{17} \mathrm{ClN}_{2} \mathrm{O}\left(\mathrm{M}^{+}\right)$384.1029, found 384.1027; MS (EI, 70 eV): $\mathrm{m} / \mathrm{z}(\%)=384(18)\left[\mathrm{M}^{+}\right], 356$ (12), 293 (14), 242 (30), 241 (21), 240 (56), 234 (100), 175 (14), 149 (13), 115 (12), 105 (38), 104 (50), 77 (38).

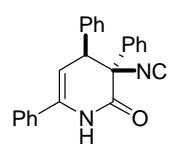

Dihydropyridone 20. According to General Procedure I, reaction between phosphonate 1, n-BuLi, benzonitrile, benzaldehyde and isocyanoacetate 14, followed by column chromatography ( $c$-hexane:EtOAc $=9: 1 \rightarrow 7: 3)$, afforded $20(343 \mathrm{mg}, 98 \%)$ as a yellow foam. Slow crystallisation from EtOAc/pentane afforded the pure product as pale yellow crystals. Mp 174-176 ${ }^{\circ} \mathrm{C}$ (decomposes); ${ }^{1} \mathrm{H}$ NMR $(250 \mathrm{MHz}$, $\left.\mathrm{CDCl}_{3}\right): \delta(\mathrm{ppm}) 8.34(\mathrm{~s}, 1 \mathrm{H}), 7.47-7.19(\mathrm{~m}, 15 \mathrm{H}), 5.72(\mathrm{dd}, J=4.4,1.2 \mathrm{~Hz}, 1 \mathrm{H}) ; 4.32(\mathrm{~d}, J=4.5 \mathrm{~Hz}, 1 \mathrm{H}) ;{ }^{13} \mathrm{C} \mathrm{NMR}(63 \mathrm{MHz}, \mathrm{CDCl}): \delta(\mathrm{ppm}) 165.2(\mathrm{C})$, $162.4(\mathrm{C}), 137.0(\mathrm{C}), 135.6(\mathrm{C}), 135.2(\mathrm{C}), 133.4(\mathrm{C}), 129.6(\mathrm{CH}), 129.3(2 \times \mathrm{CH}), 129.1(2 \times \mathrm{CH}), 129.0(\mathrm{CH}), 128.5(2 \times \mathrm{CH}), 128.4(2 \times \mathrm{CH}), 128.3(\mathrm{CH})$, $126.3(2 \times \mathrm{CH}), 125.2(2 \times \mathrm{CH}), 105.1(\mathrm{CH}), 70.4(\mathrm{C}), 51.4(\mathrm{CH})$; IR (neat): 2129 (s), $1715(\mathrm{~s}), 1683(\mathrm{~s}), 1495(\mathrm{~s}), 1455$ (s), 1265 (s); HRMS (EI, 70 eV): calculated for $\mathrm{C}_{24} \mathrm{H}_{18} \mathrm{~N}_{2} \mathrm{O}\left(\mathrm{M}^{+}\right)$350.1419, found 350.1405; MS (EI, $\left.70 \mathrm{eV}\right): \mathrm{m} / \mathrm{z}(\%)=208$ (73), 207 (100), 206 (94), 191 (31), 179 (21), 165 (11), 147 (13), 131 (30), 119 (29), 115 (25), 105 (86), 104 (62), 103 (35). Crystals suitable for X-ray crystal structure determination were obtained by the slow diffusion of pentane into a saturated solution of $\mathbf{1 8}$ in THF.

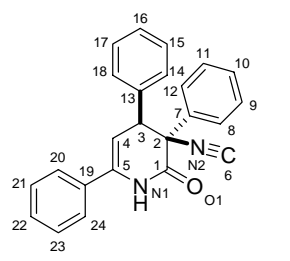

Crystallographic Data for 20. $\mathrm{C}_{24} \mathrm{H}_{18} \mathrm{~N}_{2} \mathrm{O}, \mathrm{FW}=350.40$, colourless triangular plate, $0.51 \times 0.36 \times 0.12 \mathrm{~mm}^{3}$, triclinic, $\mathrm{P} \overline{1}$ (no. 2), $a=7.2566(2), b=8.17547(18), c=16.3566(7) \AA ̊ ., \alpha=92.313(1), \beta=100.627(1), \gamma=103.381(1)^{\circ}, V=924.32(5) \AA^{3}, Z=2, D_{x}=$ $1.259 \mathrm{~g} / \mathrm{cm}^{3}, \mu=0.08 \mathrm{~mm}^{-1} .23518$ Reflections were measured on a Nonius Kappa CCD diffractometer with rotating anode (graphite monochromator, $\lambda=0.71073 \AA)$ up to a resolution of $(\sin \theta / \lambda)_{\max }=0.65 \AA^{-1}$ at a temperature of $110 \mathrm{~K}$. The reflections were corrected for absorption and scaled on the basis of multiple measured reflections with the program SADABS ${ }^{[1]}(0.85-1.00$ correction range). 4258 Reflections were unique $\left(\mathrm{R}_{\mathrm{int}}=0.0259\right)$. The structure was solved with Direct Methods ${ }^{[2}$ and refined with SHELXL-97 ${ }^{[3}$ against $\mathrm{F}^{2}$ of all reflections. Non hydrogen atoms were refined with anisotropic displacement parameters. All hydrogen atoms were located in the difference Fourier map. The N-H hydrogen atom was refined freely with isotropic displacement parameters; all other hydrogen atoms were refined with a riding model. 248 Parameters were refined with no restraints. R1/wR2 [I > 2 $\sigma(\mathrm{I})$ ]: 0.0437/0.1015. R1/wR2 [all reflections]: 0.0579/0.1100. S $=1.026$. Residual electron density between -0.30 and 0.27 e/ $\AA^{3}$. Geometry calculations and checking for higher symmetry was performed with the PLATON program. ${ }^{[4]}$

CCDC 612759 contains the supplementary crystallographic data for this paper. These data can be obtained free of charge from The Cambridge Crystallographic Data Centre via www.ccdc.cam.ac.uk/data_request/cif. 
Table 1. Selected bond distances $(\AA)$ in the crystal structure of 20. Standard uncertainties are given in parentheses.

\begin{tabular}{llll}
\hline $\mathrm{C} 2-\mathrm{O} 7$ & $1.2248(18)$ & $\mathrm{C} 5-\mathrm{C} 6$ & $1.3394(16)$ \\
$\mathrm{N} 1-\mathrm{C} 2$ & $1.3448(19)$ & $\mathrm{C} 6-\mathrm{N} 1$ & $1.4174(18)$ \\
$\mathrm{C} 2-\mathrm{C} 3$ & $1.5601(16)$ & $\mathrm{C} 3-\mathrm{N} 8$ & $1.4501(16)$ \\
$\mathrm{C} 3-\mathrm{C} 4$ & $1.5605(16)$ & $\mathrm{N} 8-\mathrm{C} 9$ & $1.1580(19)$ \\
$\mathrm{C} 4-\mathrm{C} 5$ & $1.5068(17)$ & & \\
\hline
\end{tabular}

Table 2. Selected bond angles $\left(^{\circ}\right)$ in the crystal structure of $\mathbf{2 0}$. Standard uncertainties are given in parentheses.

\begin{tabular}{llll}
\hline N1-C2-C3 & $116.13(12)$ & C3-C2-O7 & $120.23(12)$ \\
C2-C3-C4 & $108.38(9)$ & N1-C2-O7 & $123.59(11)$ \\
C3-C4-C5 & $109.84(10)$ & C2-C3-N8 & $104.32(10)$ \\
C4-C5-C6 & $121.70(12)$ & C4-C3-N8 & $109.20(10)$ \\
C5-C6-N1 & $118.12(12)$ & C3-N8-C9 & $177.77(15)$ \\
C6-N1-C2 & $125.46(11)$ & & \\
\hline
\end{tabular}

Table 3. Hydrogen bonding geometry in the crystal structure of 20. Standard uncertainties are given in parentheses. ${ }^{a}$

\begin{tabular}{ccccc}
\hline D-H...A & D-H $(\AA)$ & H...A $(\AA)$ & D....A $(\AA)$ & D-H...A $\left({ }^{\circ}\right)$ \\
\hline N1-H1...O7 $7^{\text {i }}$ & $0.887(19)$ & $2.009(19)$ & $2.8821(14)$ & $167.9(16)$ \\
& & & \\
${ }^{a}$ Symmetry operation i: 1-x, 1-y, 1-z.. & & & \\
\hline
\end{tabular}

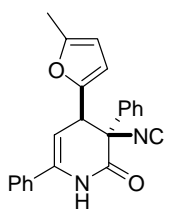

Dihydropyridone 24. According to General Procedure I, reaction between phosphonate 1, $n$-BuLi, benzonitrile, 5-methylfurfural and isocyanoacetate 14, followed by column chromatography ( $c$-hexane:EtOAc $=9: 1 \rightarrow 4: 6$ ), afforded 24 as a yellow foam $(128 \mathrm{mg}, 36 \%) .{ }^{1} \mathrm{H}$ NMR $\left(250 \mathrm{MHz}, \mathrm{CDCl}_{3}\right): \delta(\mathrm{ppm}) 8.17(\mathrm{~s}, 1 \mathrm{H}), 7.55-7.38(\mathrm{~m}, 10 \mathrm{H}), 6.18(\mathrm{~d}, J=2.6 \mathrm{~Hz}, 1 \mathrm{H}), 5.90(\mathrm{~s}, 1 \mathrm{H}), 5.57(\mathrm{~d}, J=5.4 \mathrm{~Hz}, 1 \mathrm{H}), 4.40$ (d, $J=5.4 \mathrm{~Hz}, 1 \mathrm{H}), 2.20(\mathrm{~s}, 3 \mathrm{H}) ;{ }^{13} \mathrm{C}$ NMR (63 MHz): $\delta(\mathrm{ppm}) 165.2(\mathrm{C}), 161.5(\mathrm{C}), 152.7(\mathrm{C}), 147.2(\mathrm{C}), 137.2(\mathrm{C}), 135.0(\mathrm{C}), 133.3(\mathrm{C})$, $129.5(\mathrm{CH}), 129.2(\mathrm{CH}), 128.9(2 \times \mathrm{CH}), 128.7(2 \times \mathrm{CH}), 125.9(2 \times \mathrm{CH}), 125.1(2 \times \mathrm{CH}), 110.0(\mathrm{CH}), 106.4(\mathrm{CH}), 102.1(\mathrm{CH}), 69.1(\mathrm{C}), 45.1(\mathrm{CH}), 13.4(\mathrm{CH})$; IR (neat): 2128 (s), 1693 (s), 1449 (s); HRMS (EI, $70 \mathrm{eV}$ ): calculated for $\mathrm{C}_{23} \mathrm{H}_{18} \mathrm{~N}_{2} \mathrm{O}_{2}$ (M $\left.\mathrm{M}^{+}\right)$354.1368, found 354.1356; MS (EI, $70 \mathrm{eV}$ ): m/z (\%) = 354 (42) $\left[\mathrm{M}^{+}\right], 326(12), 312$ (30), 234 (79), 212 (100), 197 (98), 168 (22), 153 (8), 141 (17), 115 (15), 77 (23).

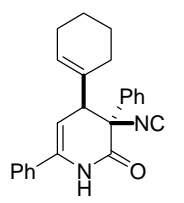

Dihydropyridone 30. According to General Procedure I, reaction between phosphonate 1, n-BuLi, benzonitrile, 1-cyclohexene-1carboxaldehyde and isocyanoacetate 14, followed by column chromatography ( $c$-hexane:EtOAc $=85: 15 \rightarrow 7: 3$ ), afforded $\mathbf{3 0}$ as a yellow solid (227 mg, 64\%). Mp 142-145 ${ }^{\circ} \mathrm{C}$ (decomposes); ${ }^{1} \mathrm{H}$ NMR (250 MHz, $\mathrm{CDCl}_{3}$ ): $\delta(\mathrm{ppm}) 8.21(\mathrm{~s}, 1 \mathrm{H}), 7.61-7.35$ (m, 10H), $5.83(\mathrm{br} \mathrm{s}, 1 \mathrm{H})$, $5.41(\mathrm{~d}, J=6.0 \mathrm{~Hz}, 1 \mathrm{H}), 3.62(\mathrm{~d}, J=6.0 \mathrm{~Hz}, 1 \mathrm{H}), 2.19-1.51(\mathrm{~m}, 8 \mathrm{H}) ;{ }^{13} \mathrm{C} \mathrm{NMR}\left(63 \mathrm{MHz}, \mathrm{CDCl}_{3}\right): \delta(\mathrm{ppm}) 165.9(\mathrm{C}), 160.7(\mathrm{C}), 136.3(\mathrm{C})$, $136.2(\mathrm{C}), 134.1(\mathrm{C}), 133.5(\mathrm{C}), 129.4(\mathrm{CH}), 129.1(\mathrm{CH}), 129.02(2 \times \mathrm{CH}), 129.96(\mathrm{CH}), 128.8(2 \times \mathrm{CH}), 125.7(2 \times \mathrm{CH}), 124.9(2 \times \mathrm{CH}), 103.4(\mathrm{CH}), 68.7(\mathrm{C})$, 
54.0 (CH), $25.6\left(\mathrm{CH}_{2}\right), 25.3\left(\mathrm{CH}_{2}\right), 22.7\left(\mathrm{CH}_{2}\right), 21.9\left(\mathrm{CH}_{2}\right)$; IR (neat): 2141 (s), 1691 (s); HRMS (EI, $\left.70 \mathrm{eV}\right)$ : calculated for $\mathrm{C}_{24} \mathrm{H}_{22} \mathrm{~N}_{2} \mathrm{O}\left(\mathrm{M}^{+}\right)$354.1732, found

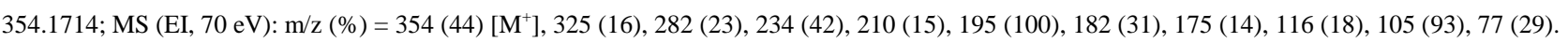

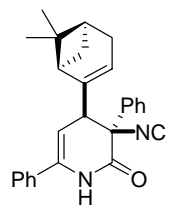

Dihydropyridone 32. According to General Procedure I, reaction between phosphonate 1, $n$-BuLi, benzonitrile, $(1 R)-(-)$-myrtenal and isocyanoacetate 14, followed by column chromatography ( $c$-hexane:EtOAc $=85: 15 \rightarrow 7: 3$ ), afforded 32 as a $1: 1$ mixture of two diasteroisomers as a yellow foam $(304 \mathrm{mg}, 77 \%) .{ }^{1} \mathrm{H}$ NMR $\left(250 \mathrm{MHz}, \mathrm{CDCl}_{3}\right): \delta(\mathrm{ppm}) 8.19(\mathrm{~s}, 1 \mathrm{H}), 8.08(\mathrm{~s}, 1 \mathrm{H}), 7.61-7.35(\mathrm{~m}, 10 \mathrm{H}+$ $10 \mathrm{H}), 5.67(\mathrm{~s}, 1 \mathrm{H}), 5.52(\mathrm{~s}, 1 \mathrm{H}), 5.41(\mathrm{~s}, 1 \mathrm{H}), 5.31(\mathrm{~s}, 1 \mathrm{H}), 3.80-3.74(\mathrm{~m}, 1 \mathrm{H}+1 \mathrm{H}), 2.49-2.06(\mathrm{~m}, 5 \mathrm{H}+5 \mathrm{H}), 1.28-1.19(\mathrm{~m}, 4 \mathrm{H}+4 \mathrm{H}), 0.89$ (s, 3H), 0.85 (s, 3H); ${ }^{13} \mathrm{C}$ NMR (63 MHz, $\left.\mathrm{CDCl}_{3}\right)$ : $\delta$ (ppm) 165.6 (C), 165.5 (C), 162.0 (C), 161.5 (C), 142.7 (C), 142.0 (C), $136.4(\mathrm{C}), 136.3(\mathrm{C}), 136.2(\mathrm{C})$, $135.7(\mathrm{C}), 133.8(\mathrm{C}), 133.6(\mathrm{C}), 129.45(\mathrm{CH}), 129.39(\mathrm{CH}), 129.1(2 \times \mathrm{CH}), 129.0(2 \times \mathrm{CH}+1 \times \mathrm{CH}), 128.7(2 \times \mathrm{CH}), 128.6(2 \times \mathrm{CH}), 126.6(\mathrm{CH}), 126.1(2 \times \mathrm{CH})$, $125.7(2 \times \mathrm{CH}), 125.04(2 \times \mathrm{CH}), 124.98(2 \times \mathrm{CH}), 124.2(\mathrm{CH}), 123.7(\mathrm{CH}), 104.2(\mathrm{CH}), 102.5(\mathrm{CH}), 69.0(\mathrm{C}), 68.5(\mathrm{C}), 53.0(\mathrm{CH}), 51.1(\mathrm{CH}), 47.2(\mathrm{CH}), 43.6$ $(\mathrm{CH}), 40.4(\mathrm{CH}), 40.2(\mathrm{CH}), 37.9(\mathrm{C}), 37.8(\mathrm{C}), 32.3\left(\mathrm{CH}_{2}\right), 31.9\left(\mathrm{CH}_{2}\right), 31.7\left(2 \times \mathrm{CH}_{2}\right), 26.2\left(2 \times \mathrm{CH}_{3}\right), 21.7\left(\mathrm{CH}_{3}\right), 21.4\left(\mathrm{CH}_{3}\right)$; IR $($ neat $): 2141(\mathrm{~s}), 1697(\mathrm{~s})$, 1693 (s), 1451 (s), 1254 (s), 1214 (s); HRMS (EI, $70 \mathrm{eV}$ ): calculated for $\mathrm{C}_{27} \mathrm{H}_{26} \mathrm{~N}_{2} \mathrm{O}$ (M+) 394.20.45, found 394.2026; MS (EI, $70 \mathrm{eV}$ ): m/z (\%) = 394 (14) $\left[\mathrm{M}^{+}\right], 351$ (11), 325 (16), 235 (14), 175 (17), 130 (14), $116(50), 105$ (29), 90 (35), 83 (100), 77 (24), 59 (14).

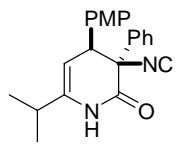

Dihydropyridone 34. According to General Procedure I, reaction between phosphonate 1, $n$-BuLi, $i$-butyronitrile, $p$-anisaldehyde and isocyanoacetate 14, followed by column chromatography ( $c$-hexane:EtOAc $=7: 3)$, afforded $\mathbf{3 4}(197 \mathrm{mg}, 57 \%)$ as a yellow foam. ${ }^{1} \mathrm{H}$ NMR $\left(400 \mathrm{MHz}, \mathrm{CDCl}_{3}\right): \delta(\mathrm{ppm}) 7.95(\mathrm{~s}, 1 \mathrm{H}), 7.40-7.34(\mathrm{~m}, 5 \mathrm{H}), 6.99(\mathrm{~d}, J=8.7 \mathrm{~Hz}, 2 \mathrm{H}), 6.75(\mathrm{~d}, J=8.8 \mathrm{~Hz}, 2 \mathrm{H}), 5.10(\mathrm{dd}, J=2.7,1.0 \mathrm{~Hz}$, 1H), $4.02(\mathrm{~d}, J=4.2 \mathrm{~Hz}, 1 \mathrm{H}), 3.75(\mathrm{~s}, 3 \mathrm{H}), 2.40(\mathrm{~m}, 1 \mathrm{H}), 1.11(\mathrm{~d}, J=6.3 \mathrm{~Hz}, 6 \mathrm{H}) ;{ }^{13} \mathrm{C} \mathrm{NMR}\left(50 \mathrm{MHz}, \mathrm{CDCl}_{3}\right): \delta(\mathrm{ppm}) 165.5(\mathrm{C}) 161.2(\mathrm{C}), 159.2(\mathrm{C}), 142.6$ (C), $135.5(\mathrm{C}), 130.2(2 \times \mathrm{CH}), 128.2(\mathrm{CH}), 127.84(2 \times \mathrm{CH}), 127.76(\mathrm{C}), 126.2(2 \times \mathrm{CH}), 113.6(2 \times \mathrm{CH}), 101.6(\mathrm{CH}), 70.6(\mathrm{C}), 55.2(\mathrm{CH}), 49.9(\mathrm{CH}), 31.2$ $(\mathrm{CH}), 20.0\left(\mathrm{CH}_{3}\right), 20.0\left(\mathrm{CH}_{3}\right)$; IR (KBr): 2127 (m), $1708(\mathrm{~s}), 1513(\mathrm{~m}), 1458(\mathrm{~s}), 1250(\mathrm{~m})$; HRMS (EI, $\left.70 \mathrm{eV}\right)$ : calculated for $\mathrm{C}_{22} \mathrm{H}_{22} \mathrm{~N}_{2} \mathrm{O}_{2}\left(\mathrm{M}^{+}\right) 346.1618$, found 346.1678; MS (EI, $70 \mathrm{eV}): \mathrm{m} / \mathrm{z}(\%)=346(100)\left[\mathrm{M}^{+}\right], 331(9), 303(18), 202(80)$.

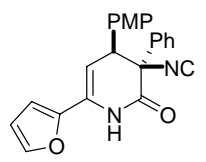

Dihydropyridone 36. According to General Procedure I, reaction between phosphonate 1, $n$-BuLi, 2-furonitrile, $p$-anisaldehyde and isocyanoacetate 14, followed by column chromatography $(c$-hexane:EtOAc $=85: 15 \rightarrow 7: 3)$, afforded 36 as a red oil $(282 \mathrm{mg}, 76 \%) .{ }^{1} \mathrm{H}$ $\operatorname{NMR}\left(250 \mathrm{MHz}, \mathrm{CDCl}_{3}\right): \delta(\mathrm{ppm}) 9.41(\mathrm{~s}, 1 \mathrm{H}), 7.52-7.38(\mathrm{~m}, 6 \mathrm{H}), 7.12(\mathrm{~d}, J=8.4 \mathrm{~Hz}, 2 \mathrm{H}), 6.80(\mathrm{~d}, J=8.5 \mathrm{~Hz}, 2 \mathrm{H}), 6.69(\mathrm{~d}, J=3.4$ $\left.\mathrm{Hz}, 1 \mathrm{H}), 6.39(\mathrm{~d}, J=1.6 \mathrm{~Hz}, 1 \mathrm{H}), 5.87(\mathrm{~d}, J=4.8 \mathrm{~Hz}, 1 \mathrm{H}), 4.29(\mathrm{~d}, J=4.8 \mathrm{~Hz}, 1 \mathrm{H}), 3.76(\mathrm{~s}, 3 \mathrm{H}) ;{ }^{13} \mathrm{C} \mathrm{NMR}(63 \mathrm{MHz}, \mathrm{CDCl})_{3}\right) \delta(\mathrm{ppm}) 165.5(\mathrm{C}), 162.0(\mathrm{C})$, $159.49(\mathrm{C}), 146.3(\mathrm{C}), 143.1(\mathrm{CH}), 135.4(\mathrm{C}), 130.3(2 \times \mathrm{CH}), 129.0(\mathrm{CH}), 128.6(2 \times \mathrm{CH}), 128.0(\mathrm{C}), 127.3(\mathrm{C}), 126.2(2 \times \mathrm{CH}), 113.8(2 \times \mathrm{CH}), 111.7(\mathrm{CH})$, $107.3(\mathrm{CH}), 102.5(\mathrm{CH}), 70.6(\mathrm{C}), 55.1\left(\mathrm{CH}_{3}\right), 50.2(\mathrm{CH})$; IR (neat): $2129(\mathrm{~m}), 1699(\mathrm{~s}), 1511(\mathrm{~s}), 1256(\mathrm{~m})$; HRMS (EI, 70 eV): calculated for $\mathrm{C}_{23} \mathrm{H}_{18} \mathrm{~N}_{2} \mathrm{O}_{3}$

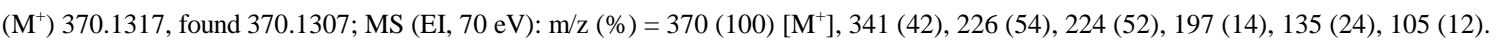

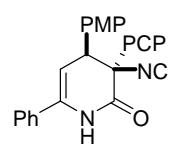

Dihydropyridone 38. According to General Procedure I, reaction between phosphonate 1, $n$-BuLi, benzonitrile, $p$-anisaldehyde and isocyanoacetate 37, followed by column chromatography ( $c$-hexane:EtOAc $=8: 2 \rightarrow 6: 4)$, afforded 38 as a yellow solid $(249 \mathrm{mg}, 60 \%)$. Mp $187-190{ }^{\circ} \mathrm{C}$ (decomposes); ${ }^{1} \mathrm{H}$ NMR $\left(250 \mathrm{MHz}, \mathrm{DMSO}-d_{6}\right): \delta(\mathrm{ppm}) 10.62(\mathrm{~s}, 1 \mathrm{H}), 7.60(\mathrm{~d}, J=8.7 \mathrm{~Hz}, 2 \mathrm{H}), 7.51-7.48(\mathrm{~m}, 4 \mathrm{H}), 7.40-7.37$ $(\mathrm{m}, 3 \mathrm{H}), 7.21(\mathrm{~d}, J=8.6 \mathrm{~Hz}, 2 \mathrm{H}), 6.90(\mathrm{~d}, J=8.6 \mathrm{~Hz}, 2 \mathrm{H}), 5.70(\mathrm{~d}, J=5.5 \mathrm{~Hz}, 1 \mathrm{H}), 4.55(\mathrm{~d}, J=5.6 \mathrm{~Hz}, 1 \mathrm{H}), 3.31(\mathrm{~s}, 3 \mathrm{H}) ;{ }^{13} \mathrm{C} \mathrm{NMR}\left(63 \mathrm{MHz}, \mathrm{CDCl}_{3}\right): \delta$ (ppm) $164.5(\mathrm{C}), 163.1(\mathrm{C}), 159.7(\mathrm{C}), 136.9(\mathrm{C}), 135.0(\mathrm{C}), 133.6(2 \times \mathrm{C}), 130.4(2 \times \mathrm{CH}), 129.9(\mathrm{CH}), 129.3(2 \times \mathrm{CH}), 128.7(2 \times \mathrm{CH}), 128.0(2 \times \mathrm{CH}), 127.0(\mathrm{C})$, $125.2(2 \times \mathrm{CH}), 113.9(2 \times \mathrm{CH}), 105.8(\mathrm{CH}), 70.2(\mathrm{C}), 55.3\left(\mathrm{CH}_{3}\right), 50.9(\mathrm{CH})$. IR (neat): $2126(\mathrm{~s}), 1733(\mathrm{~s}), 1716(\mathrm{~s})$; HRMS (EI, $\left.70 \mathrm{eV}\right)$ : calculated for 
$\mathrm{C}_{25} \mathrm{H}_{19} \mathrm{ClN}_{2} \mathrm{O}_{2}\left(\mathrm{M}^{+}\right)$414.1135, found 414.1141; MS (EI, $\left.70 \mathrm{eV}\right): \mathrm{m} / \mathrm{z}(\%)=414$ (8) [M+1, 296 (82), 251 (31), 248 (56), 236 (41), 221 (35), 195 (52), 152 (62), 137 (100), 135 (68), 105 (54), 77 (41), 57 (27).

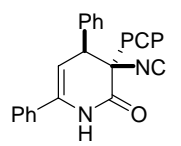

Dihydropyridone 39. According to General Procedure I, reaction between phosphonate 1, n-BuLi, benzonitrile, benzaldehyde and isocyanoacetate $\mathbf{3 7}$, followed by column chromatography ( $c$-hexane:EtOAc $=8: 2 \rightarrow 7: 3$ ), afforded 39 as a yellow solid (231 mg, $60 \%$ ). Mp $150-154{ }^{\circ} \mathrm{C}$ (decomposes); ${ }^{1} \mathrm{H}$ NMR $\left(400 \mathrm{MHz}, \mathrm{DMSO}-d_{6}\right): \delta(\mathrm{ppm}) 10.66(\mathrm{~s}, 1 \mathrm{H}), 7.60(\mathrm{~d}, J=8.5 \mathrm{~Hz}, 2 \mathrm{H}), 7.54-7.49(\mathrm{~m}, 2 \mathrm{H}), 7.50(\mathrm{~d}, J=$ $8.7 \mathrm{~Hz}, 2 \mathrm{H}), 7.40-7.38(\mathrm{~m}, 3 \mathrm{H}), 7.36-7.28(\mathrm{~m}, 5 \mathrm{H}), 5.73(\mathrm{~d}, J=5.4 \mathrm{~Hz}, 1 \mathrm{H}), 4.61(\mathrm{~d}, J=5.4 \mathrm{~Hz}, 1 \mathrm{H}), 3.31(\mathrm{~s}, 3 \mathrm{H}) ;{ }^{13} \mathrm{C} \mathrm{NMR}\left(100 \mathrm{MHz}, \mathrm{DMSO}-d_{6}\right): \delta(\mathrm{ppm})$ $164.0(\mathrm{C}), 161.0(\mathrm{C}), 137.5(\mathrm{C}), 136.0(\mathrm{C}), 134.9(\mathrm{C}), 133.7(\mathrm{C}), 133.3(\mathrm{C}), 129.2(2 \times \mathrm{CH}), 129.0(\mathrm{CH}), 128.58(2 \times \mathrm{CH}), 128.55(2 \times \mathrm{CH}), 128.3(2 \times \mathrm{CH}), 128.2$ $(2 \times \mathrm{CH}), 128.0(\mathrm{CH}), 125.6(2 \times \mathrm{CH}), 104.8(\mathrm{CH}), 69.6(\mathrm{C}), 48.5(\mathrm{CH}) ; \mathrm{IR}(\mathrm{KBr}): 2125(\mathrm{~m}), 1693(\mathrm{~s}) ; \mathrm{HRMS}\left(\mathrm{EI}, 70\right.$ eV): calculated for $\mathrm{C}_{24} \mathrm{H}_{17} \mathrm{ClN}{ }_{2} \mathrm{O}\left(\mathrm{M}^{+}\right)$ 384.1029, found 384.1034; MS (EI, $70 \mathrm{eV}): \mathrm{m} / \mathrm{z}(\%)=384(27)\left[\mathrm{M}^{+}\right], 356(17), 268(63), 206(100)$.

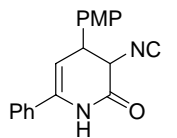

Dihydropyridone 41. 1.2 equiv. of $n$-BuLi (1.6 M solution in hexane) were added at $-78{ }^{\circ} \mathrm{C}$ to a stirred solution of phosphonate 1 ( 1 mmol, $153 \mathrm{mg})$ in THF $(5 \mathrm{~mL})$. After stirring at $-78^{\circ} \mathrm{C}$ for $1.5 \mathrm{~h}$ benzonitrile $(1.1 \mathrm{mmol}, 113 \mathrm{mg})$ was added and the mixture was then stirred at -78 ${ }^{\circ} \mathrm{C}$ for $45 \mathrm{~min}$., at $-40{ }^{\circ} \mathrm{C}$ for $1 \mathrm{~h}$ and at $-5^{\circ} \mathrm{C}$ for $30 \mathrm{~min}$. Then, $p$-anisaldehyde $(1.1 \mathrm{mmol}, 150 \mathrm{mg})$ was added and, after stirring at $-5^{\circ} \mathrm{C}$ for $30 \mathrm{~min}$., the mixture was allowed to warm to rt and stirred for $1.5 \mathrm{~h}$. The reaction mixture was slowly added to a solution of isocyanoacetate 40 (1.1 mmol, 109 $\mathrm{mg}$ ) in $0.5 \mathrm{~mL}$ THF via a syringe. The reaction mixture was stirred for $18 \mathrm{~h}$ at $\mathrm{rt}$ and concentrated in vacuo. Purification of the crude product by column chromatography $(c$-hexane:EtOAc $=17: 3 \rightarrow 6: 4)$ afforded 41 as a pale yellow foam $(97 \mathrm{mg}, 32 \%$, trans:cis $=63: 37) .{ }^{1} \mathrm{H} \mathrm{NMR}\left(400 \mathrm{MHz}, \mathrm{CDCl}{ }_{3}, 45{ }^{\circ} \mathrm{C}\right): \delta$ (ppm) $7.71($ br s, $1 \mathrm{H}+1 \mathrm{H}), 7.50-7.40(\mathrm{~m}, 5 \mathrm{H}+5 \mathrm{H}), 7.29-7.23(\mathrm{~m}, 2 \mathrm{H}+2 \mathrm{H}), 6.93(\mathrm{~d}, J=8.7 \mathrm{~Hz}, 2 \mathrm{H}), 6.89(\mathrm{~d}, J=8.7 \mathrm{~Hz}, 2 \mathrm{H}), 5.66(\mathrm{dd}, J=5.8,1.5 \mathrm{~Hz}$, $1 \mathrm{H}), 5.47(\mathrm{dd}, J=2.7,1.7 \mathrm{~Hz}, 1 \mathrm{H}), 4.77(\mathrm{~d}, J=6.7 \mathrm{~Hz}, 1 \mathrm{H}), 4.45(\mathrm{~d}, J=12.4 \mathrm{~Hz}, 1 \mathrm{H}), 4.21-4.02(\mathrm{~m}, 1 \mathrm{H}+1 \mathrm{H}), 3.81(\mathrm{~s}, 3 \mathrm{H}), 3.79(\mathrm{~s}, 3 \mathrm{H}) ;{ }^{13} \mathrm{C} \mathrm{NMR}(100$ $\mathrm{MHz}, \mathrm{CDCl}_{3}, 45^{\circ} \mathrm{C}$ ): $\delta$ (ppm) $163.2(\mathrm{C}), 162.9(\mathrm{C}), 162.8$ (C), $162.0(\mathrm{C}), 159.9$ (C), 159.7 (C), 137.5 (C), 137.1 (C), 133.7 (C), $133.6(\mathrm{C}), 130.5$ (C), 129.8 $(2 \times \mathrm{CH}), 129.7(2 \times \mathrm{CH}), 129.20(\mathrm{CH}+\mathrm{CH}), 129.18(2 \times \mathrm{CH}), 129.1(2 \times \mathrm{CH}), 126.6(\mathrm{C}), 125.2(2 \times \mathrm{CH}), 125.1(2 \times \mathrm{CH}), 114.7(2 \times \mathrm{CH}), 114.5(2 \times \mathrm{CH}), 105.4$ $(\mathrm{CH}), 104.3(\mathrm{CH}), 60.2(\mathrm{CH}), 58.4(\mathrm{CH}), 55.4\left(\mathrm{CH}_{3}\right), 55.3\left(\mathrm{CH}_{3}\right), 45.4(\mathrm{CH}), 42.7(\mathrm{CH})$; IR (neat): $2152(\mathrm{~s}), 1697(\mathrm{~s}), 1512(\mathrm{~m}) ; \mathrm{HRMS}(\mathrm{EI}, 70$ eV): calculated for $\mathrm{C}_{19} \mathrm{H}_{16} \mathrm{~N}_{2} \mathrm{O}_{2}\left(\mathrm{M}^{+}\right)$304.1212, found 304.1207; MS (EI, $\left.70 \mathrm{eV}\right): \mathrm{m} / \mathrm{z}(\%)=303$ (64). 289 (100), 257 (41), 170 (57).

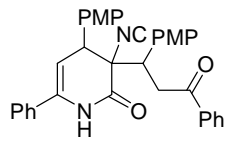

Dihydropyridone 43. According to General Procedure I, reaction between phosphonate 3, $n$-BuLi, benzonitrile, $p$-anisaldehyde and isocyanoacetate 40, followed by column chromatography ( $c$-hexane:EtOAc $=8: 2 \rightarrow 7: 3)$, afforded 41 as a yellow foam $(58 \mathrm{mg}, 19 \%$, trans:cis $=67: 33)$ together with 43 as a yellow oil $(98 \mathrm{mg}, 18 \%) .{ }^{1} \mathrm{H}$ NMR $\left(400 \mathrm{MHz}, \mathrm{CDCl}_{3}\right): \delta(\mathrm{ppm}) 7.92-7.89(\mathrm{~m}, 2 \mathrm{H}), 7.58-7.46$ (m, 4H), 7.45-7.29 (m, 7H), 7.00-6.98 (m, 2H), 6.91-6.87 (m, 2H), 6.80-6.77 (m, 2H), 5.57 (dd, J=6.8, $1.6 \mathrm{~Hz}, 1 \mathrm{H}), 4.26(\mathrm{t}, J=5.5 \mathrm{~Hz}, 1 \mathrm{H}), 3.80(\mathrm{~s}, 3 \mathrm{H})$, $3.74(\mathrm{~s}, 3 \mathrm{H}), 3.73(\mathrm{~d}, J=5.5 \mathrm{~Hz}, 2 \mathrm{H}), 3.34(\mathrm{~d}, J=6.8 \mathrm{~Hz}, 1 \mathrm{H}) ;{ }^{13} \mathrm{C} \mathrm{NMR}\left(100 \mathrm{MHz}, \mathrm{CDCl}_{3}\right): \delta(\mathrm{ppm}) 196.8(\mathrm{C}), 165.6(\mathrm{C}), 162.7(\mathrm{C}), 159.5(\mathrm{C}), 159.4(\mathrm{C})$, $137.1(\mathrm{C}), 136.5(\mathrm{C}), 133.5(\mathrm{C}), 133.1(\mathrm{CH}), 130.0(2 \times \mathrm{CH}), 129.6(\mathrm{CH}), 129.4(\mathrm{C}), 129.1(2 \times \mathrm{CH}), 128.4(2 \times \mathrm{CH}), 128.0(2 \times \mathrm{CH}), 126.9(\mathrm{C}), 125.2(4 \times \mathrm{CH})$, $114.1(2 \times \mathrm{CH}), 114.0(2 \times \mathrm{CH}), 103.6(\mathrm{CH}), 74.2(\mathrm{C}), 55.1\left(\mathrm{CH}_{3}\right), 55.1\left(\mathrm{CH}_{3}\right), 45.9(\mathrm{CH}), 42.7(\mathrm{CH}), 40.5(\mathrm{CH}) ; \mathrm{IR}(\mathrm{neat}): 2135(\mathrm{~m}), 1693(\mathrm{~s}), 1683(\mathrm{~s}), 1511$ (s); HRMS (EI, $70 \mathrm{eV}$ ): calculated for $\mathrm{C}_{35} \mathrm{H}_{30} \mathrm{~N}_{2} \mathrm{O}_{4}\left(\mathrm{M}^{+}\right)$542.2206, found 542.2199; MS (EI, $\left.70 \mathrm{eV}\right): \mathrm{m} / \mathrm{z}(\%)=542(0.5), 303(42), 238(100), 161(88)$.

(1) Sheldrick, G.M. (1999). SADABS: Area-Detector Absorption Correction, v2.10, University of Göttingen, Germany.

(2) Sheldrick, G.M. (1997). SHELXS-97. Program for crystal structure solution. University of Göttingen, Germany.

(3) Sheldrick, G.M. (1997). SHELXL-97. Program for crystal structure refinement. University of Göttingen, Germany.

(4) Spek, A.L. J. Appl. Cryst. 2003, 36, 7-13. 

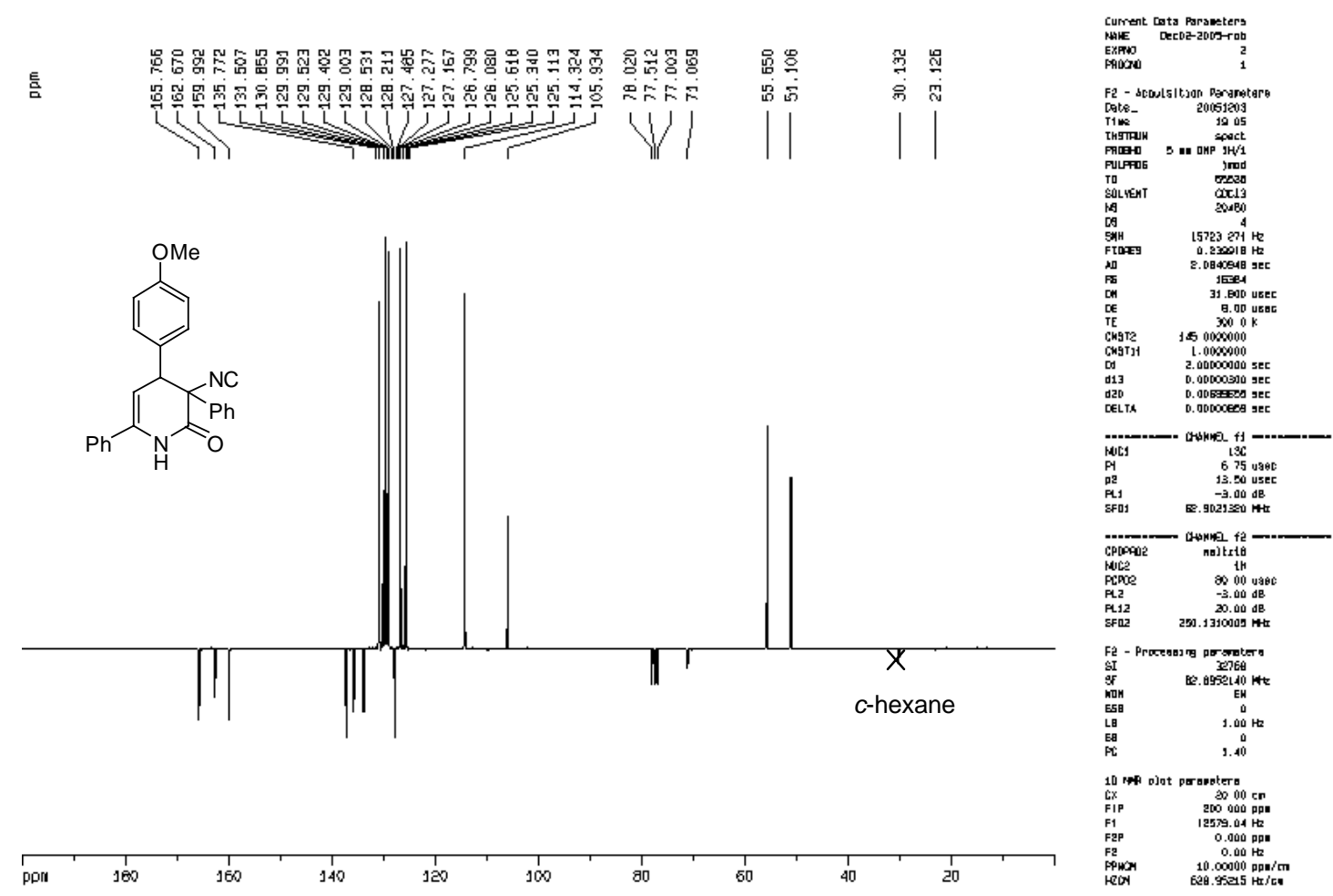

HeN E20.
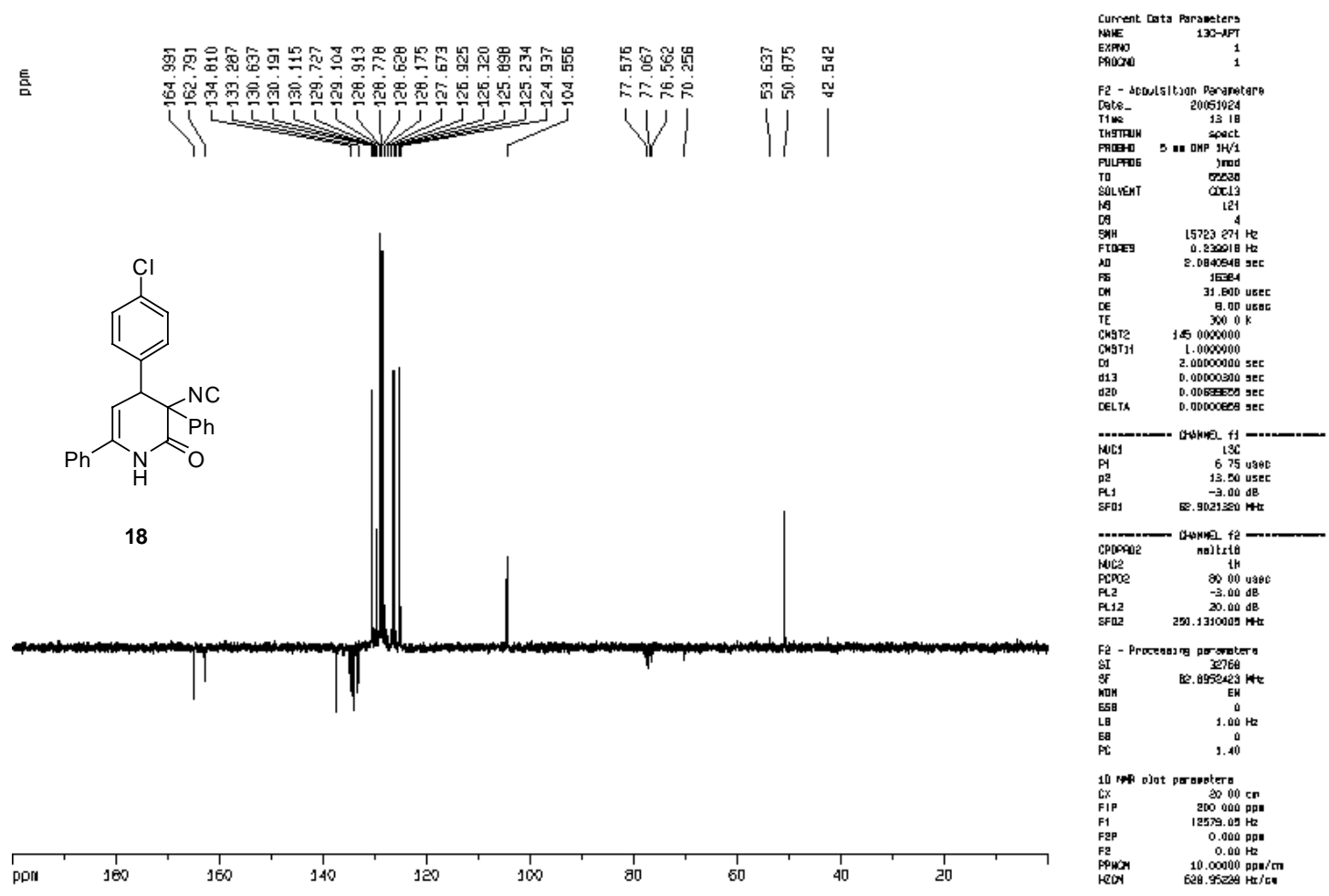

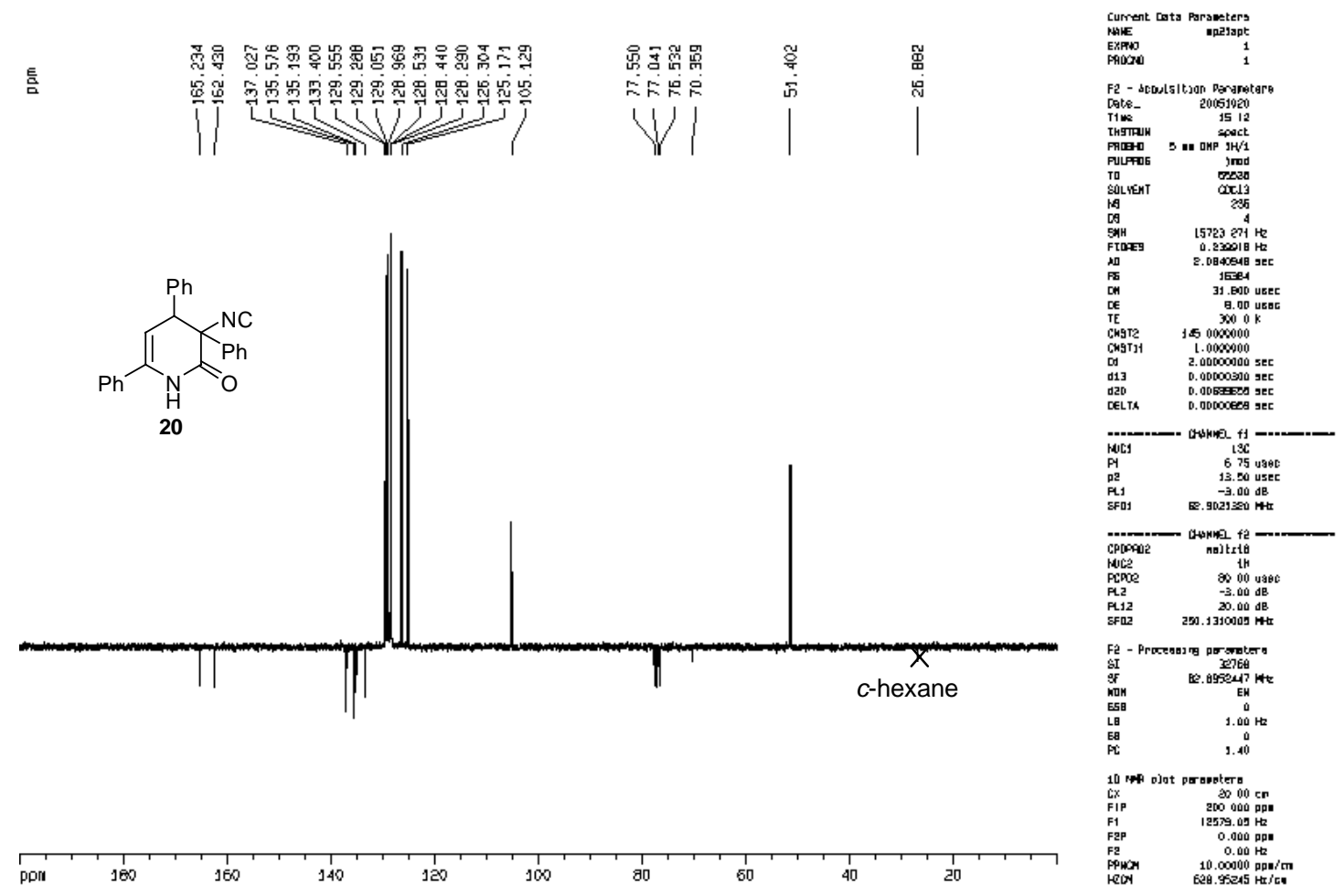

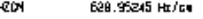

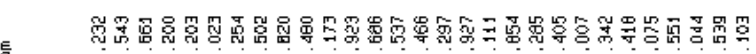

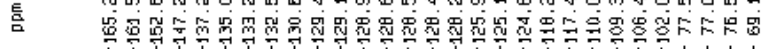

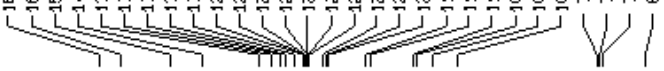

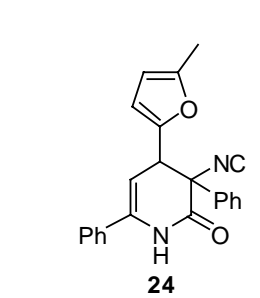

pon
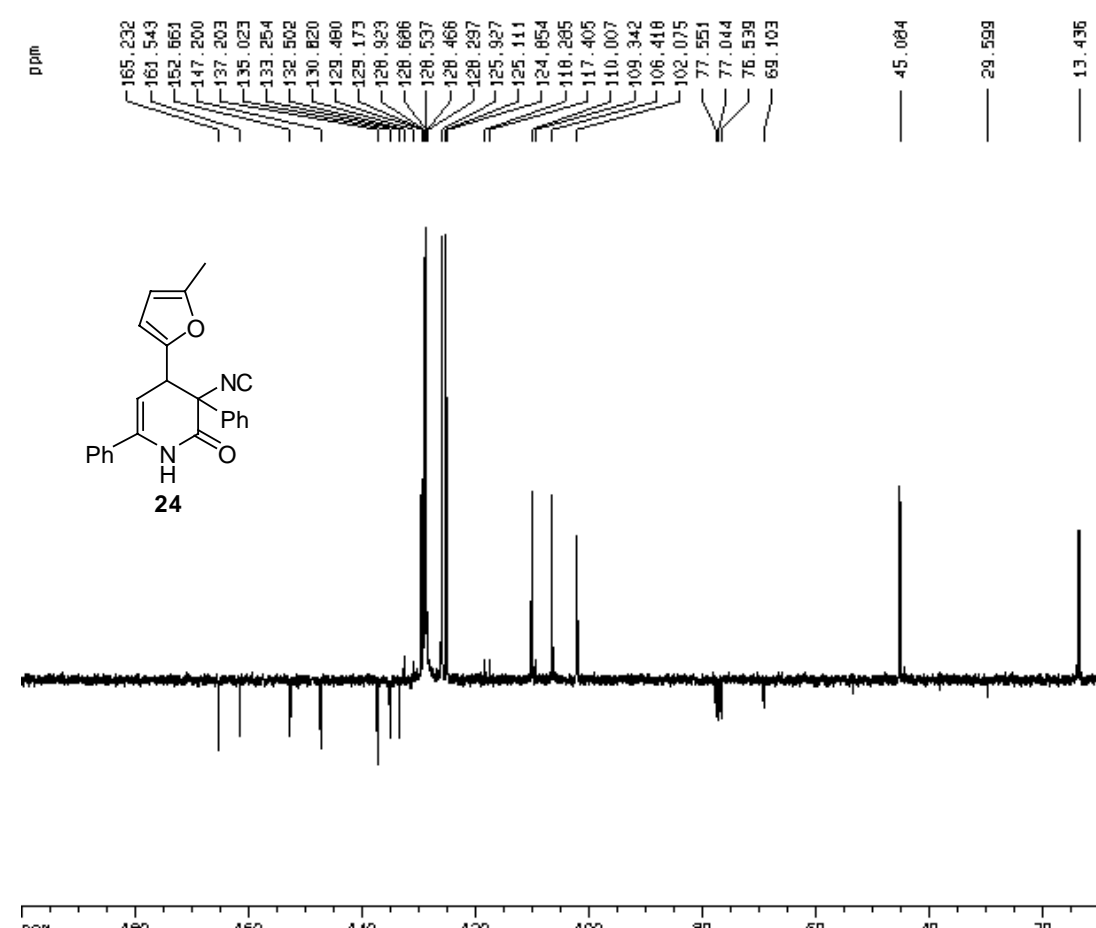
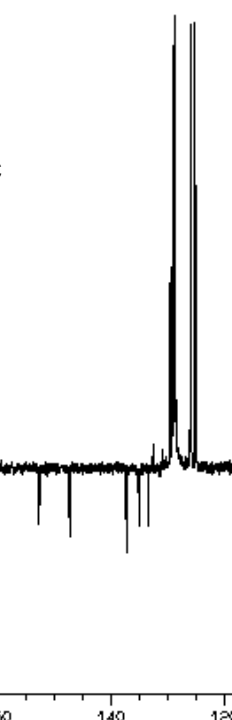

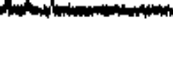

I
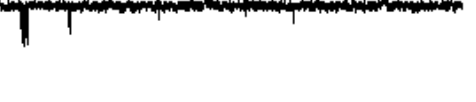

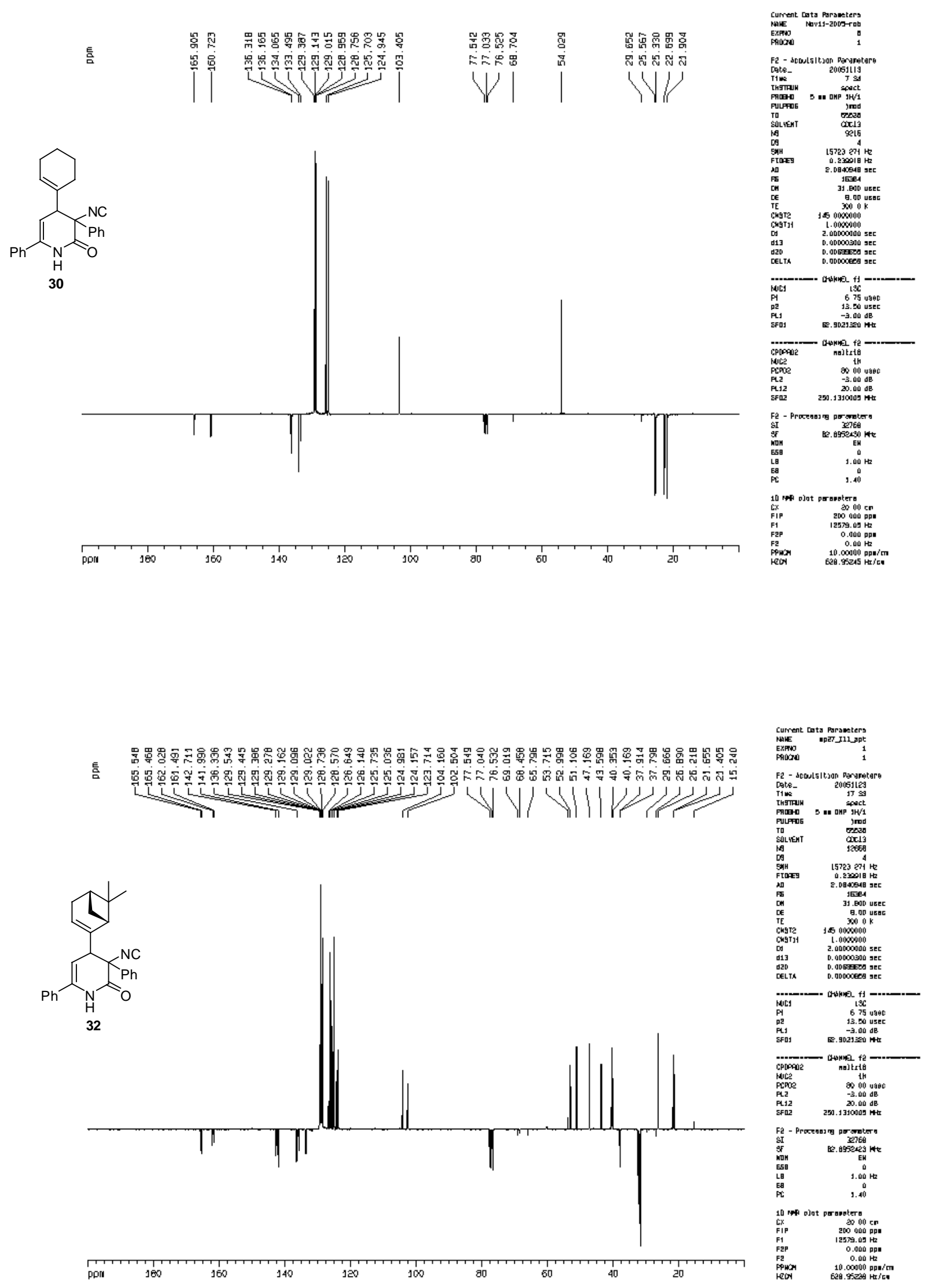

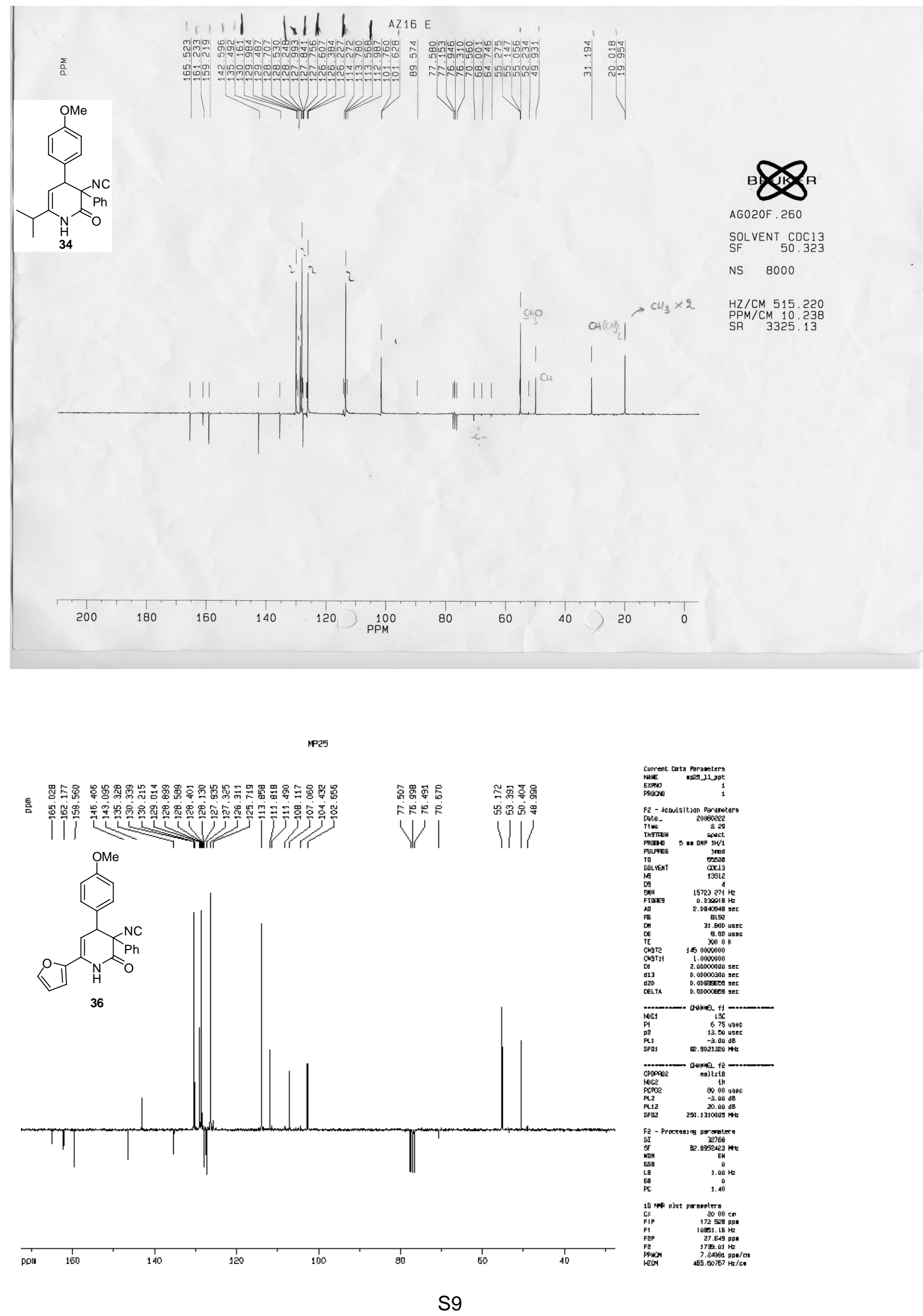
तो।<smiles>COc1ccc(C2C=C(c3ccccc3)NC(=O)C2(C)N)cc1</smiles>
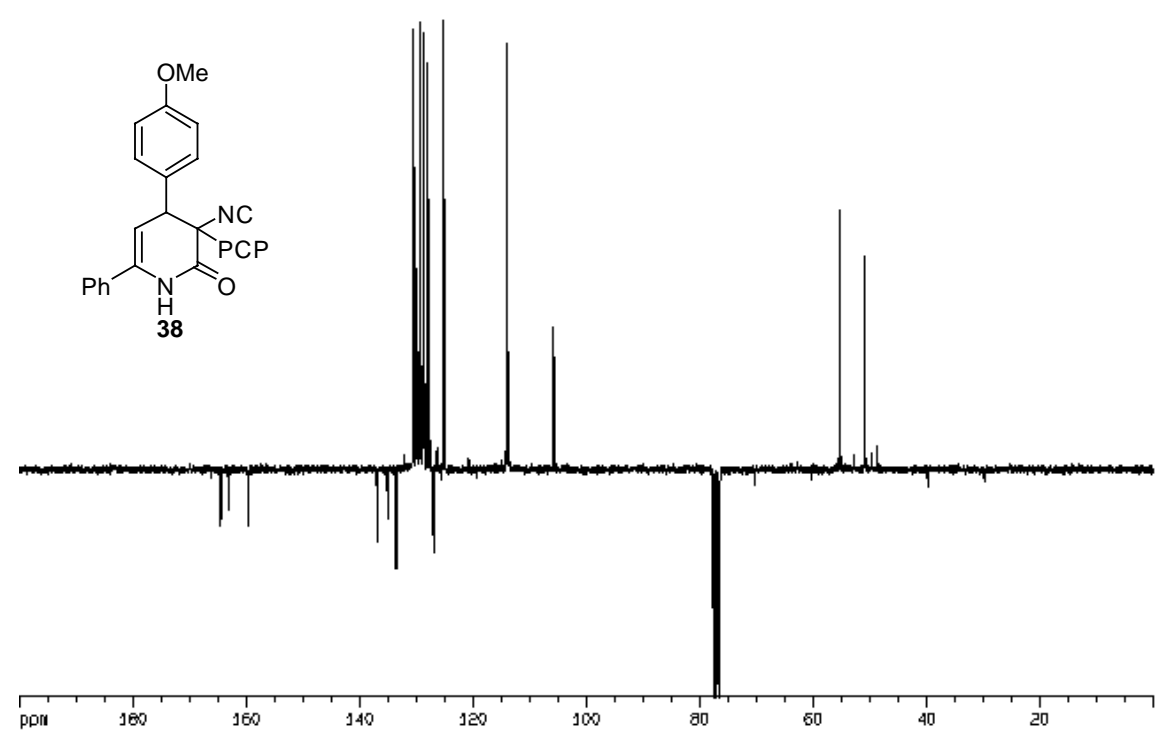

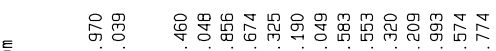

言
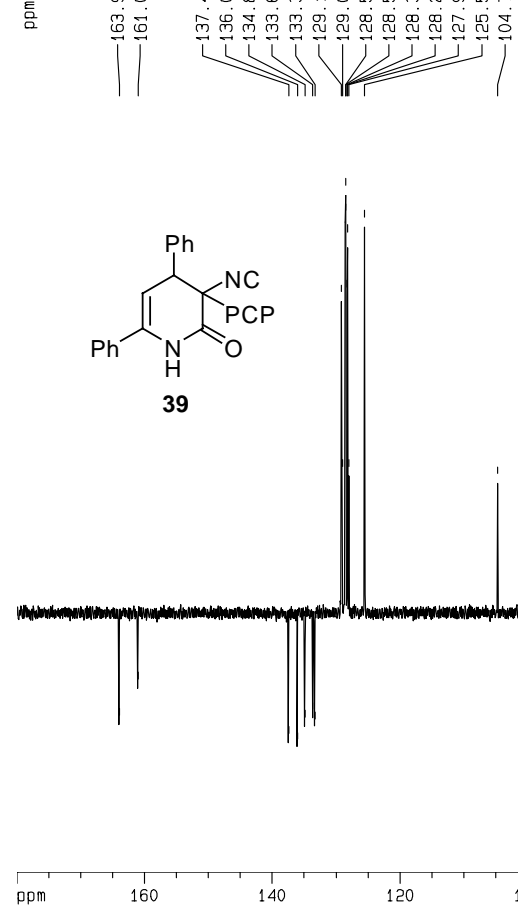

ppm

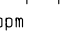

160
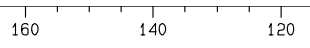

120
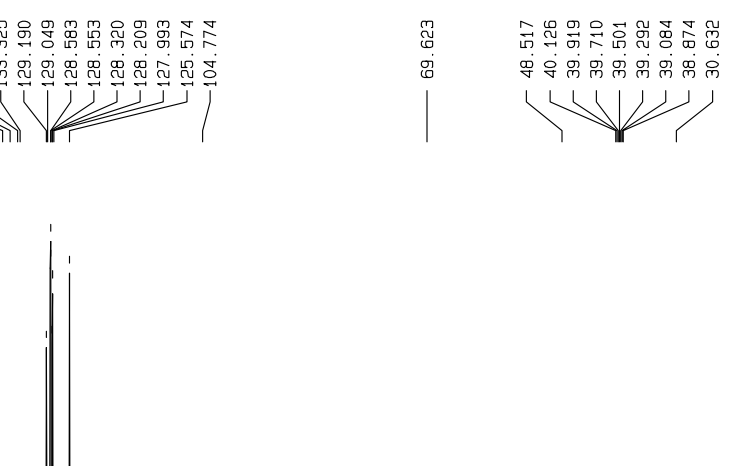

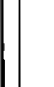

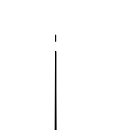

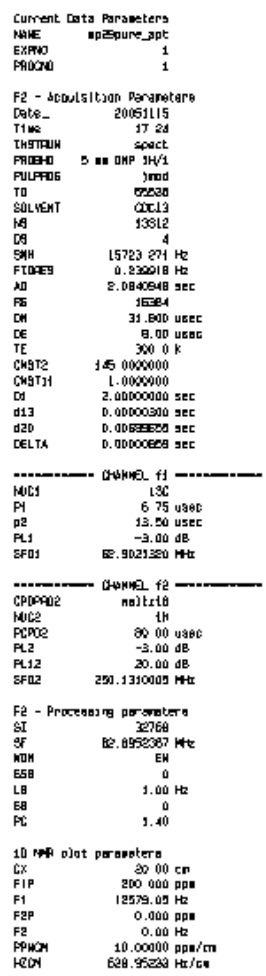

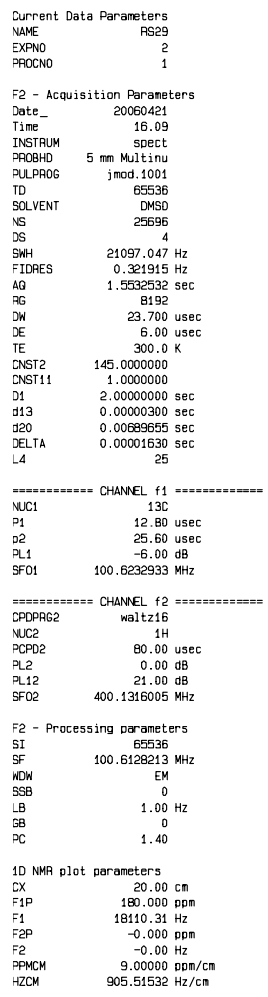




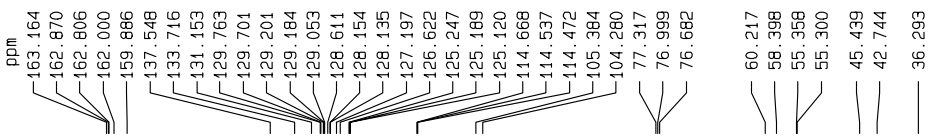<smiles>COc1ccc(C2C=C(c3ccccc3)NC(=O)C2[N+](=O)[O-])cc1</smiles>

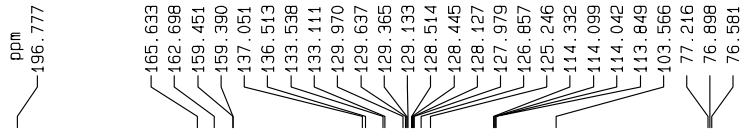

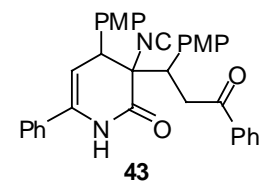

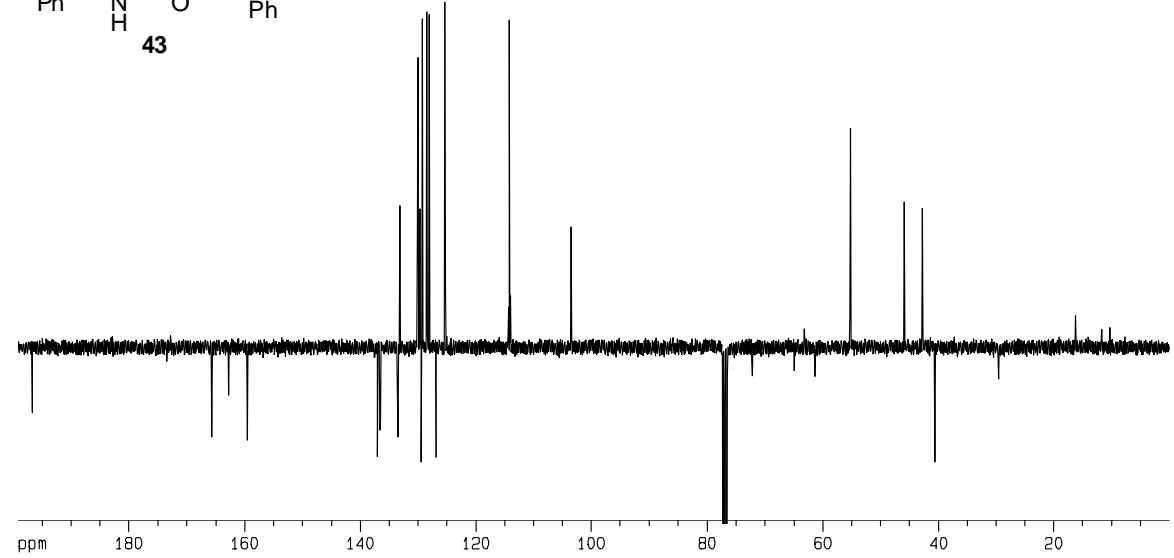

\section{只}

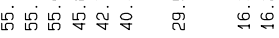
$\forall 111 \%$

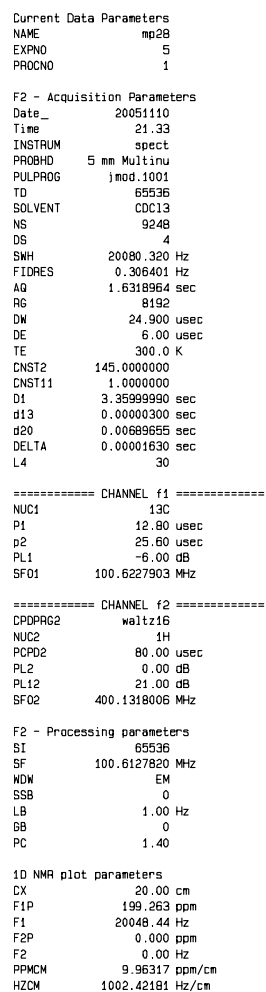



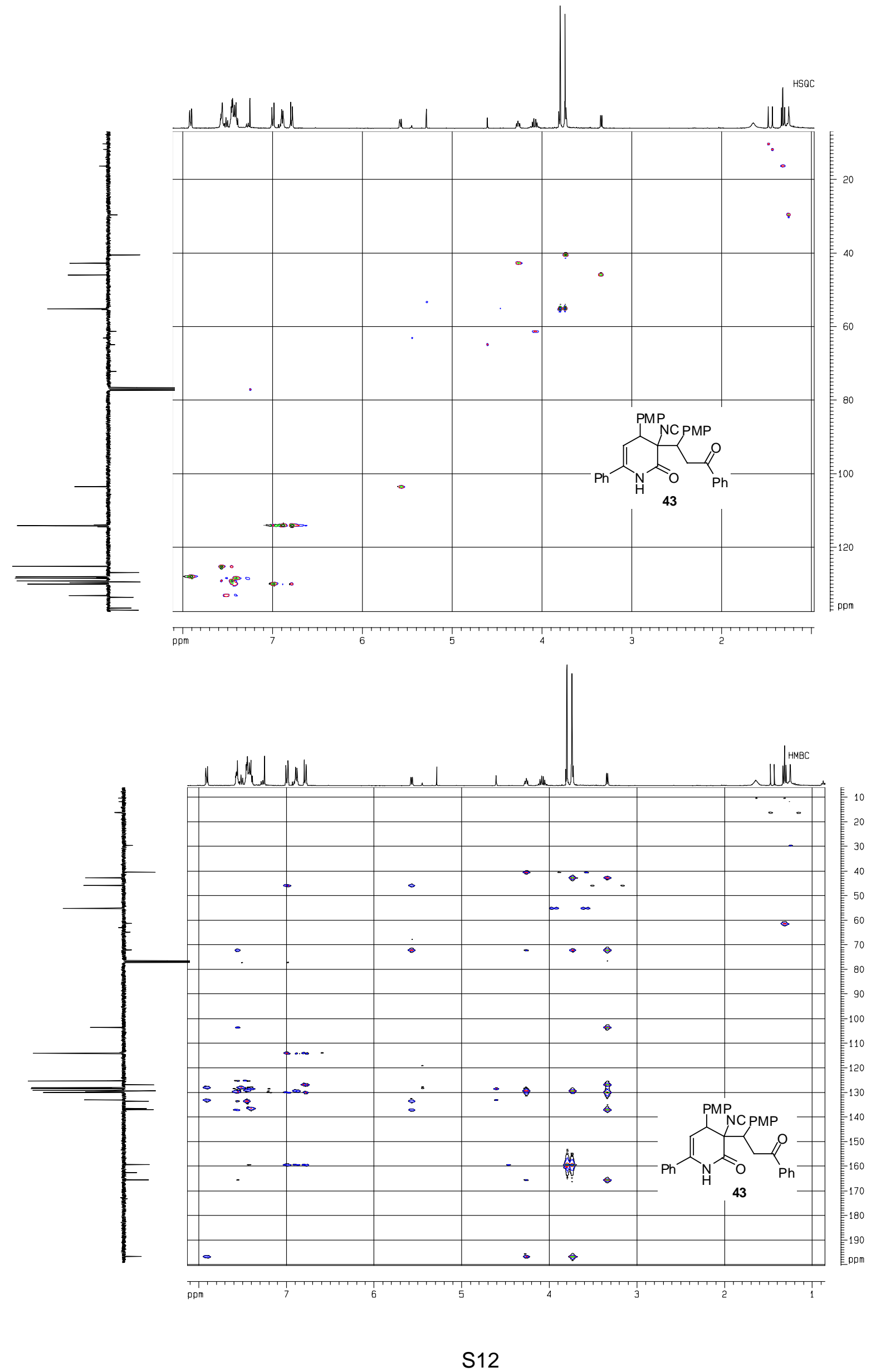